\title{
A Novel Hybrid Robust Control Design Method for F-16 Aircraft Longitudinal Dynamics
}

\author{
Vi H. Nguyen and Thanh T. Tran \\ Faculty of Engineering, Vietnamese-German University, Le Lai, Thu Dau Mot, Binh Duong, Vietnam \\ Correspondence should be addressed to Thanh T. Tran; thanh.tt@vgu.edu.vn
}

Received 4 June 2020; Revised 20 August 2020; Accepted 10 September 2020; Published 22 September 2020

Academic Editor: Baltazar Aguirre Hernandez

Copyright (C) 2020 Vi H. Nguyen and Thanh T. Tran. This is an open access article distributed under the Creative Commons Attribution License, which permits unrestricted use, distribution, and reproduction in any medium, provided the original work is properly cited.

\begin{abstract}
This paper presents a hybrid robust control design method for a third-order lower-triangular model of nonlinear dynamic systems in the presence of disturbance. In this paper, a novel control design is presented systematically to synthesize a robust nonlinear feedback controller, called backstepping sliding mode control (BSMC), for the proposed system by a combined approach of backstepping design and sliding mode control. In this approach, a family of the "sliding surface" is introduced in state transformations. Then, a smooth switching function of the sliding surface is introduced and enforced to include in virtual feedbacks and a real control law from the control selection phrases of the backstepping design loop. The achieved control method proves a well-tracking command with asymptotic stability, provides a robustness in the presence of uncertainties, and eliminates completely a chattering phenomenon. The application of flight-path angle control corresponding to the longitudinal dynamics of a high-performance F-16 aircraft simulation model is implemented. Under some assumptions, full nonlinear longitudinal dynamics is reformed into a lower-triangular system for a direct application to formulate a control law. A closed-loop system is achieved for in-flight simulation with different flight profiles for a comparison of the existing methods. Also, an external disturbance on different loading/unloading conditions in flight is applied to verify and validate robustness of the proposed control method.
\end{abstract}

\section{Introduction}

A standard design method of the flight control system for nonlinear aircraft dynamic systems is based on gain scheduling from linear control system designs. In this method, a linearized model of the nonlinear flight dynamic system at key operating points within the flight envelope is achieved. From those selected points, linear controllers are designed and then combined continuously as the aircraft flies from one operating point to another. In [1], it is indicated that actual system performance and stability can be significantly different from design results due to linearized nonlinearities. Therefore, a direct application of a nonlinear flight system should be addressed for a new generation of flight control design [2-4]. However, these works neglected the effects of uncertainties that can lead to instability and poor performance of an aircraft system due to complex variations in aerodynamic models. Recently, robust control design methods for nonlinear aircraft flight dynamics have been addressed by many researchers [5-7]. Twenty-eight uncertain parameters are used to model aircraft motion in nonlinear longitudinal dynamics [5]. Then, a genetic algorithm is used to search a feasible design coefficient space that is evaluated by Monte Carlo for stability and performance robustness. A better approach in robust control methods $[6,7]$ is proposed to improve the handling qualities across the specified flight envelope without the use of gain scheduling. In this approach, the control parameters of dynamic inversion are designed to minimize the probability of violating design specifications and so provide a better design with good robustness in stability and performance subject to modeling uncertainties. However, mentioned control design methods are mostly focused on design of control parameters that are not fully able to adapt internal dynamics in order to ensure the stability and performance robustness. Thus, a new type of the robust controller, in 
which a robust property for a nonlinear dynamic system must be included, is necessary to improve stability and performance of aircraft longitudinal dynamics.

In recent years, backstepping or recursive control design for a strict-feedback form of nonlinear dynamic systems is introduced firstly by authors [1] and is applied extensively for flight control of the nonlinear flight dynamic system [8-13]. In approaches [8-12], a full nonlinear dynamic system is transformed directly or indirectly into a strict-feedback form or a lower-triangular model for applicability via assumptions or state transformations. The achieved model will be divided into subsystems for control objectives. By considering each subsystem in relation with interconnected subsystems, a virtual feedback control is selected from satisfying the requirement of Lyapunov's theorem. After a series of control design for subsystems, a nonlinear feedback controller is obtained. By numerical simulation and experimental study, the backstepping control method can prove a very good performance and stability if system parameter modeling is accurate. On the other hand, the method might lead to a poor quality across the full flight envelope due to uncertain system parameters. Thus, a better approach [13] is proposed to improve the performance in this situation. In this design, an integrator with a tracking error is introduced and added into the control law via feedback selection. The achieved control system can improve robust performance and stability in the presence of disturbance, but time response of the flightpath angle or the attack angle is slower than that of the traditional methods. This behavior or robustness should be improved in designing the control system for a highperformance aircraft system.

Sliding mode control (SMC) was proposed firstly and applied by Slotine [14]. This method provides a robust control technique to deal with unmodeling dynamics or uncertain system parameters for nonlinear dynamic systems and has been widely used and extended for flight control design [15-21]. A linearized model for longitudinal dynamics is achieved for applying the sliding mode control design [17]. The achieved control method is simulated on the nonlinear flight longitudinal model of the F-16 aircraft. An extra controller of the integral sliding mode control allocation scheme [18], which is active if faults occurs, is built around a traditional controller. Although numerical simulation indicates that the proposed control shows good results for nominal and fault/failure conditions, a guideline on how it works or a stability proof is a concern in the paper. In order to solve for fuzzy stochastic systems subjected to matched/ mismatched uncertainties, Wang et al. [20] propose a new method to remove these assumptions and present a new integral sliding mode control (ISMC) method. As discussed above, it is clear that each work has some advantage and some limitation, but mostly based on the traditional control design method. So, the question arises if there exists a systematic approach to formulate a robust nonlinear feedback controller that can inherit advances of the backstepping control and robust aspects of a sliding mode control design. This paper will provide a novel solution to deal with the proposed challenge by a combined design of the backstepping method and sliding mode control. In this proposal, a family of the "sliding surface" is introduced in state transformations. Then, the sliding surface and a smooth switching function are enforced to include in virtual feedbacks and a real control law from control selection phrases. Due to the inheritance of the properties of the backstepping control and sliding mode control, the achieved control method proves a well-tracking command with asymptotic stability, provides a robustness in the presence of uncertainties, and eliminates completely a chattering phenomenon. The application of flight-path angle control of a high-performance F-16 aircraft model is implemented to show advance of the proposed control method.

This paper is organized as follows: a hybrid controller formulation for a general class of a third-order lower-triangular model of a nonlinear dynamic system is presented in Section 2. Study of flight-path angle control of longitudinal dynamics of the F-16 aircraft mode is investigated to show applicability in Section 3. A numerical simulation of the full nonlinear F-16 aircraft model is implemented and compared to the integrator-backstepping control method; finally, conclusion and discussions are presented in the end of the paper.

\section{A Hybrid Controller Formulation for a Single- Input Single-Output (SISO) Third-Order Lower-Triangular Model of Nonlinear Dynamic Systems}

In this section, a problem formulation is considered firstly. Secondly, a new control design method is presented for formulating a backstepping sliding mode control (BSMC) law for the (SISO) third-order lower-triangular form of the nonlinear dynamic system. Some important remarks are given in the end of the section.

2.1. Problem Formulation. Consider an SISO third-order lower-triangular model [1] of nonlinear dynamics systems as

$$
\begin{aligned}
& \dot{x}_{1}=f_{1}\left(x_{1}\right)+g_{1}\left(x_{1}\right) x_{2}, \\
& \dot{x}_{2}=f_{2}\left(x_{1}, x_{2}\right)+g_{2}\left(x_{1}, x_{2}\right) x_{3}, \\
& \dot{x}_{2}=f_{2}\left(x_{1}, x_{2}, x_{3}\right)+g_{2}\left(x_{1}, x_{2}, x_{3}\right) u,
\end{aligned}
$$

where the unknown valued functions $\hat{f}_{i}(i=1,2,3)$ and $\hat{g}_{i}(i=1,2,3)$ are approximated as known functions $f_{i}(i=1,2,3)$ and $g_{i}(i=1,2,3)$, respectively, in this paper. This approximation is based on working condition and experience for a specific system. $x_{i}(i=1,2,3) \in R$ are the state variables, and the functions $f_{i}(i=1,2,3) \in R$ are known real-valued, continuous, and differentiable. The functions $g_{i}(i=1,2,3) \in R$ are known real-valued, continuous, differentiable, and invertible. The variable $u \in R$ is the control input.

The objective of this research is to synthesize a robust control law for systems (1)-(3) such that the outputs $y=$ $h(x)=x_{1}$ track well the desired value or command, $x_{d}$, 
asymptotically, where $x_{d}$ is a triple-differentiable function in time. Also, a global asymptotic stability is achieved with no or acceptably small overshoot in the presence of the model parameter errors and external disturbances.

\subsection{Formulation of Backstepping Sliding Mode Control Law}

Theorem 1. There exists coordinate transformations (4), (5), and (6) and virtual feedbacks $\alpha_{1}$ and $\alpha_{2}$ in (5) and (6), respectively, and real control $u$ in (7):

$$
\begin{aligned}
e_{1}(t) & =x_{1}-x_{d}, \\
e_{2}(t) & =x_{2}-\alpha_{1}\left(x_{1}, x_{d}, \dot{x}_{d}, p_{1}\right), \\
e_{3}(t) & =x_{3}-\alpha_{2}\left(x_{1}, x_{2}, x_{d}, \dot{x}_{d}, p_{1}, p_{2}\right), \\
u & =K\left(x_{1}, x_{2}, x_{3}, x_{d}, \dot{x}_{d}, \ddot{x}_{d}, p_{1}, p_{2}, p_{3}\right) .
\end{aligned}
$$

Such that systems (1)-(3) can be transformed into a stable linear decoupling system in terms of a new set of state variables $e_{1}, e_{2}$, and $e_{3}$ as

$$
\begin{aligned}
& \dot{e}_{1}(t)=-p_{1} e_{1}(t) \tanh \left(\lambda e_{1}(t)\right), \\
& \dot{e}_{2}(t)=-p_{2} e_{2}(t) \tanh \left(\lambda e_{2}(t)\right), \\
& \dot{e}_{3}(t)=-p_{3} e_{3}(t) \tanh \left(\lambda e_{3}(t)\right),
\end{aligned}
$$

where $p_{1}, p_{2}, p_{3}$, and $\lambda$ are the strict positive values and $\tanh (\cdot)$ is a hyperbolic tangent function and defined as $\tanh (\sigma)=\left(e^{\sigma}-e^{-\sigma}\right) /\left(e^{\sigma}+e^{-\sigma}\right)$.

The following is a proof for Theorem 1 .

The proof of Theorem 1 consists of three steps. Coordinate transformations are introduced firstly. Then, virtual feedbacks and real control can be selected in terms of recursive design approach [11]. By doing so, systems (1)-(3) can be reformed into systems (8)-(10).

Step 1. Taking derivative of equation (4) in association with equation (1) results in

$$
\dot{e}_{1}(t)=f_{1}\left(x_{1}\right)+g_{1}\left(x_{1}\right) x_{2}-\dot{x}_{d}
$$

In Lyapunov's approach, a virtual control law $\alpha_{1}\left(x_{1}, x_{d}, \dot{x}_{d}, p_{1}\right):=x_{2}$ can be selected such that the tracking error $e_{1}(t)$ in equation (11) converges asymptotically to zero with small or no overshoot. The virtual controller can be chosen from satisfying the condition in which time differentiation of a control Lyapunov function (CLF) [1], $L_{1}\left(e_{1}(t)\right)$, is less than or equal to a negative definitive function, $w\left(e_{1}(t)\right)$. In fact, a CLF is selected as

$$
L_{1}\left(e_{1}(t)\right)=\frac{1}{2} e_{1}(t)^{2} \text {. }
$$

Combining the result from time differentiation of the CLF, $L\left(e_{1}(t)\right)$, and equation (11) achieves

$$
\dot{L}_{1}\left(e_{1}(t)\right)=e_{1}(t) \dot{e}_{1}(t)=e_{1}(t)\left\{f_{1}\left(x_{1}\right)+g_{1}\left(x_{1}\right) x_{2}-\dot{x}_{d}\right\} \text {. }
$$

Then, the virtual control $\alpha_{1}$ can be obtained from enforcing $\dot{L}_{1}\left(e_{1}(t)\right)$ to be a negative definitive function, or

$$
\begin{aligned}
-p_{1} \tanh \left(\lambda e_{1}(t)\right)= & f_{1}\left(x_{1}\right)+g_{1}\left(x_{1}\right) x_{2}-\dot{x}_{d}, \\
\alpha_{1}\left(x_{1}, x_{d}, \dot{x}_{d}, p_{1}\right)= & g_{1}^{-1}\left(x_{1}\right)\left\{-p_{1} \tanh \left(\lambda\left(x_{1}-x_{d}\right)\right)\right. \\
& \left.-f_{1}\left(x_{1}\right)+\dot{x}_{d}\right\} .
\end{aligned}
$$

Substituting equation (14) into equation (13) results in

$$
\begin{aligned}
\dot{L}_{1}\left(e_{1}(t)\right) & =-p_{1} e_{1}(t) \tanh \left(\lambda e_{1}(t)\right) \\
& :=-w_{1}\left(e_{1}(t)\right)<0 \forall e_{1}(t) \neq 0 .
\end{aligned}
$$

Note that $w_{1}\left(e_{1}(t)\right)=p_{1} e_{1}(t) \tanh \left(\lambda e_{1}(t)\right)$ is a positive definitive function. Thus, $L_{1}\left(e_{1}(t)\right)$ is a negative definitive function. This means that an achieved virtual control $\alpha_{1}\left(x_{1}, x_{d}, \dot{x}_{d}, p_{1}\right)$ assures to make tracking error tend to zero.

Step 2. By choosing feedback (14) and state transformations $(4)$ and (5), systems (1)-(2) are resynthesized in $\left(e_{1}, e_{2}\right)$ coordinates as follows:

$$
\begin{aligned}
& \dot{e}_{1}(t)=-p_{1} \tanh \left(\lambda e_{1}(t)\right), \\
& \dot{e}_{2}(t)=f_{2}\left(e_{1}+x_{d}, e_{2}+\alpha_{1}\right)+g_{2}\left(e_{1}+x_{d}, e_{2}+\alpha_{1}\right) x_{3}-\dot{\alpha}_{1} .
\end{aligned}
$$

Noting that the functions $f_{1}$ and $\alpha_{1}$ are only functions of states and command. Thus, the term $\dot{\alpha}_{1}$ can be represented explicitly in variables of $x_{1}, x_{2}, x_{d}, \dot{x}_{d}, \ddot{x}_{d}, p_{1}$, and $\lambda$, and $\dot{\alpha}_{1}$ is calculated in the following equation:

$$
\dot{\alpha}_{1}=\frac{\partial \alpha_{1}}{\partial x_{1}} f_{1}+\frac{\partial \alpha_{1}}{\partial x_{d}} \dot{x}_{d}+\frac{\partial \alpha_{1}}{\partial \dot{x}_{d}} \ddot{x}_{d} .
$$

As a similar approach, a virtual control $\alpha_{2}$ : $=x_{3}$ and CLF $L_{2}\left(e_{1}(t), e_{2}(t)\right)$ are selected to ensure that systems (16)-(17) are stabilized at origin, i.e.,

$$
L_{2}\left(e_{1}(t), e_{2}(t)\right)=L_{1}\left(e_{1}(t)\right)+\frac{1}{2} e_{2}(t)^{2} .
$$

By taking derivative of equation (19) in consideration with equations (15)-(17), one gets

$$
\begin{aligned}
\dot{L}_{2}\left(e_{1}(t), e_{2}(t)\right)= & -w_{1}\left(e_{1}(t)\right)+e_{2}(t)\left\{f_{2}\left(e_{1}+x_{d}, e_{2}+\alpha_{1}\right)\right. \\
& \left.+g_{2}\left(e_{1}+x_{d}, e_{2}+\alpha_{1}\right) x_{3}-\dot{\alpha}_{1}\right\} .
\end{aligned}
$$

Then, a virtual control $\alpha_{2}$ can be obtained from enforcing $\dot{L}_{2}\left(e_{1}(t), e_{2}(t)\right)$ to be a negative definitive function, or 


$$
\begin{aligned}
-p_{2} \tanh \left(\lambda e_{2}(t)\right)= & f_{2}\left(e_{1}+x_{d}, e_{2}+\alpha_{1}\right) \\
& +g_{2}\left(e_{1}+x_{d}, e_{2}+\alpha_{1}\right) x_{3}-\dot{\alpha}_{1}, \\
\alpha_{2}\left(x_{1}, x_{2}, x_{d}, \dot{x}_{d}, \ddot{x}_{d}, p_{1}, p_{2}\right)= & g_{2}^{-1}\left(x_{1}, x_{2}\right)\left\{-p_{2} \tanh \left(\lambda e_{2}(t)\right)\right. \\
& \left.-f_{2}\left(x_{1}, x_{2}\right)+\dot{\alpha}_{1}\right\} .
\end{aligned}
$$

Substituting equation (21) into equation (20) results in

$$
\begin{aligned}
\dot{L}_{2}\left(e_{1}(t), e_{2}(t)\right)= & -p_{1} e_{1}(t) \tanh \left(\lambda e_{1}(t)\right) \\
& -p_{2} e_{2}(t) \tanh \left(\lambda e_{2}(t)\right) \\
:= & -w_{2}\left(e_{1}(t), e_{2}(t)\right)<0 \forall e_{1}(t), e_{2}(t) \neq 0 .
\end{aligned}
$$

Note that $w_{2}\left(e_{1}(t), e_{2}(t)\right)=p_{1} e_{1}(t) \tanh \left(\lambda e_{1}(t)\right)+$ $p_{2} e_{2}(t) \tanh \left(\lambda e_{2}(t)\right)$ is a positive definitive function. Thus, $\dot{L}_{2}\left(e_{1}(t), e_{2}(t)\right)$ is a negative definitive function. This means that an achieved virtual control $\alpha_{2}$ assures to make tracking error, $e_{2}(t)$, tend to zero or the first subsystem and the second subsystem [1] are interconnected with the proposed method.

Step 3. By choosing feedbacks (14) and (21) and state transformations (4)-(6), systems (1)-(3) are reformed in $\left(e_{1}, e_{2}, e_{3}\right)$ coordinates as follows:

$$
\begin{gathered}
\dot{e}_{1}(t)=-p_{1} \tanh \left(\lambda e_{1}(t)\right), \\
\dot{e}_{2}(t)=-p_{2} \tanh \left(\lambda e_{2}(t)\right), \\
\dot{e}_{3}(t)=f_{3}\left(e_{1}+x_{d}, e_{2}+\alpha_{1}, e_{3}+\alpha_{2}\right) \\
+g_{3}\left(e_{1}+x_{d}, e_{2}+\alpha_{1}, e_{3}+\alpha_{2}\right) u-\dot{\alpha}_{2} .
\end{gathered}
$$

Noting that the functions $f_{1}, f_{2}$ and $\alpha_{1}, \alpha_{2}$ are only functions of states and command. Thus, the term $\dot{\alpha}_{2}$ can be calculated and represented explicitly in variables of $x_{1}, x_{2}, x_{3}, x_{d}, \dot{x}_{d}, \ddot{x}_{d}, \ddot{x}_{d}, p_{1}, p_{2}$, and $\lambda$.

As a similar approach in Step 2, a real nonlinear feedback control, called backstepping sliding mode control (BSMC) $u=K\left(x_{1}, x_{2}, x_{3}, x_{d}, \dot{x}_{d}, \dot{x}_{d}, p_{1}, p_{2}, p_{3}\right)$ in (7), and a CLF $L_{3}\left(e_{1}(t), e_{2}(t), e_{3}(t)\right)$ are selected to ensure that systems $(26)-(28)$ are stabilized at origin $(0,0,0)$, i.e.,

$$
L_{3}\left(e_{1}(t), e_{2}(t), e_{3}(t)\right)=L_{2}\left(e_{1}(t), e_{2}(t)\right)+\frac{1}{2} e_{3}(t)^{2} .
$$

By taking derivative of equation (26) in consideration with equations (15), (23), (24), and (25), one gets

$$
\begin{aligned}
\dot{L}_{3}= & -w_{2}+e_{3}(t)\left\{f_{3}\left(e_{1}+x_{d}, e_{2}+\alpha_{1}, e_{3}+\alpha_{2}\right)\right. \\
& \left.+g_{3}\left(e_{1}+x_{d}, e_{2}+\alpha_{1}, e_{3}+\alpha_{2}\right) u-\dot{\alpha}_{2}\right\} .
\end{aligned}
$$

With the given assumption that the function $g_{3}\left(x_{1}, x_{2}, x_{3}\right)$ can be inverse, after some manipulations and return of original variables, a BSMC can be achieved as follows:

$$
\begin{aligned}
-p_{3} \tanh \left(\lambda e_{3}(t)\right)= & f_{3}\left(e_{1}+x_{d}, e_{2}+\alpha_{1}, e_{3}+\alpha_{2}\right) \\
& +g_{3}\left(e_{1}+x_{d}, e_{2}+\alpha_{1}, e_{3}+\alpha_{2}\right) u-\dot{\alpha}_{2}, \\
u:= & K=g_{3}^{-1}\left(x_{1}, x_{2}, x_{3}\right)\left\{-p_{3} \tanh \left(\lambda e_{3}(t)\right)\right. \\
& \left.-f_{3}\left(x_{1}, x_{2}\right)+\dot{\alpha}_{2}\right\} .
\end{aligned}
$$

Substituting equation (28) into equation (27) results in

$$
\begin{aligned}
\dot{L}_{3}= & -p_{1} e_{1}(t) \tanh \left(\lambda e_{1}(t)\right)-p_{2} e_{2}(t) \tanh \left(\lambda e_{2}(t)\right) \\
& -p_{3} e_{3}(t) \tanh \left(\lambda e_{3}(t)\right), \\
:= & -w_{3}\left(e_{1}(t), e_{2}(t), e_{3}(t)\right)<0 \forall e_{1}(t), e_{2}(t), e_{3}(t) \neq 0 .
\end{aligned}
$$

Note that $w_{3}=p_{1} e_{1}(t) \tanh \left(\lambda e_{1}(t)\right)+p_{2} e_{2}(t)$ $\tanh \left(\lambda e_{2}(t)\right)+p_{3} e_{3}(t) \tanh \left(\lambda e_{3}(t)\right)$ is a positive definitive function. Thus, $\dot{L}_{3}\left(e_{1}(t), e_{2}(t), e_{3}(t)\right)$ is a negative definitive function. This again ensures that the tracking error $e_{3}$ tends to origin.

Substituting (28) into (25), systems (1)-(3) in a new coordinate $e_{1}, e_{2}, e_{3}$ are rewritten as follows:

$$
\begin{aligned}
& \dot{e}_{1}(t)=-p_{1} \tanh \left(\lambda e_{1}(t)\right), \\
& \dot{e}_{2}(t)=-p_{2} \tanh \left(\lambda e_{2}(t)\right), \\
& \dot{e}_{3}(t)=-p_{3} \tanh \left(\lambda e_{3}(t)\right),
\end{aligned}
$$

where $p_{1}, p_{2}, p_{3}$, and $\lambda$ are the strict positive values.

Thus, there exists state transformations (4)-(6), virtual feedbacks (14) and (21), and real control law (28) such that systems (1)-(3) can be transformed into systems (8)-(10) or systems (30)-(32).

With the achieved control law in (28), substituting this control law into systems (1)-(3) results in a closed-loop system that is used to implement numerical simulation with different commands and initial conditions.

Remark 1. With positive values of $p_{1}, p_{2}, p_{3}$, and $\lambda$, the solutions $e_{1}(t), e_{2}(t)$, and $e_{3}(t)$ of systems (30)-(32) are stabilized at origin $(0,0,0)$ at a certain time. This means that the BSMC will enforce the outputs $y=h(x)=x_{1}$ to have a well-tracking command $x_{d}$.

Remark 2. It is observed that the tracking errors $e_{1}(t), e_{2}(t)$, and $e_{3}(t)$ play a similar role as "sliding surfaces" in sliding mode control and the smooth switch function $\tanh \left(\lambda e_{2}\right) \approx \tanh \left(\lambda\left[x_{2}+p_{1}\left(x_{1}-x_{d}\right)+\cdots\right]\right)$ plays a "similar" role as switch function sign $(\cdot)$ of the sliding surface in traditional SMC. This term will ensure the robustness of the proposed method as system parameter errors or external disturbance is present in the system.

Remark 3. If $f_{1}=0 ; g_{1}=1, f_{2}=0 ; g_{2}=1, f_{3}=f ; g_{3}=1$; and $\tanh (\lambda e):=e$, then the term $e_{2}=x_{2}-\alpha_{1}=x_{2}-\dot{x}_{d}+$ $p_{1}\left(x_{1}-x_{d}\right)$ is defined exactly as a sliding surface in the 
traditional SMC [14] and the term $-p \tanh \left(e_{2}\right)$ is similar to the corrective input $-K \operatorname{sign}(\sigma)$ in [14].

Remark 4. Noting that the hyperbolic tangent function $\tanh (\lambda \sigma)$ with a large value $\lambda$ will reach to the discontinuous sign function sign $(\cdot)$. Thus, a very large value of $\lambda$ might lead to a chattering problem. A suitable parameter of $\lambda$ must be traded off for a good performance.

Remark 5. From equations (8)-(10), it is clear that the convergence speed of the proposed control algorithm will follow the exponential rule with gains $p_{1}, p_{2}$, and $p_{3}$. The greater the gains are, the faster the convergence rate is. Besides, the convergence speed of the proposed control algorithm also depends on the function $\tanh (e)$. If you would like to gain a faster response, you can increase $\lambda$, but a trade-off between $\lambda$ and chattering issue must be considered for good performance.

\section{Flight-Path Angle Control of F-16 Aircraft Model}

In this section, the lower-triangular model of nonlinear longitudinal dynamics is introduced and explained for design suitability. An explicit BSMC law is presented by using the proposed theory in Section 2. Then, a numerical demonstration of flight-path angle control of the F-16 aircraft simulation model is implemented to show the advances, robustness, and applicability of the proposed method to flight dynamic systems as compared to the existing methods.

3.1. Problem Formulation. Consider longitudinal dynamics of the aircraft flight system as shown in Figure 1.

Assumptions on aerodynamic forces [10] and use of the relationship $\alpha=\theta-\gamma$ are applied to transform an original longitudinal motion into the lower-triangular systems (1)-(3):

$$
\begin{aligned}
& \dot{\gamma}=-g_{p} \cos \gamma+\widehat{L}_{0}-\widehat{L}_{\alpha} \gamma+\widehat{L}_{\alpha} \theta, \\
& \dot{\theta}=q, \\
& \dot{q}=\widehat{M}_{0}+\widehat{M}_{\alpha}(\theta-\gamma)+\widehat{M}_{q} q+\widehat{M}_{\delta_{E}} \delta_{E},
\end{aligned}
$$

where $g_{p}=\left(g / V_{T}\right), \widehat{L}_{0}=\left(L_{0} / m V_{T}\right), \widehat{L}_{\alpha}=\left(L_{\alpha} / m V_{T}\right), \widehat{M}_{0}=$ $\left(M_{0} / I_{y}\right), \hat{M}_{\alpha}=\left(M_{\alpha} / I_{y}\right), \widehat{M}_{q}=\left(M_{q} / I_{y}\right)$, and $\widehat{M}_{\delta_{E}}=\left(M_{\delta_{E}} l\right.$ $\left.I_{y}\right)$. Variables and parameters appearing include $V_{T}$ : aircraft velocity, $m$ : total mass of the aircraft, $\alpha$ : angle of attack, $\gamma$ : flight-path angle, $\theta$ : pitch angle, $q$ : pitch rate, $\delta_{E}$ : pitch control (elevator or horizontal vane), $I_{y}$ : inertial moment about the $y$ axis of the aircraft, $g$ : gravity, $\widehat{L}_{0}$ : effective lift contribution from sources other than $\alpha, \widehat{L}_{\alpha}$ : effective lift curve slope for $\alpha, \widehat{M}_{0}$ : effective moment contributions from sources other than $\alpha, q, \delta_{E}, \widehat{M}_{\alpha}$ and $\widehat{M}_{q}$ : effective moment contributions from sources $\alpha, q$, and $\hat{M}_{\delta_{E}}$ : effective pitch curve slope for $\delta_{E}$.

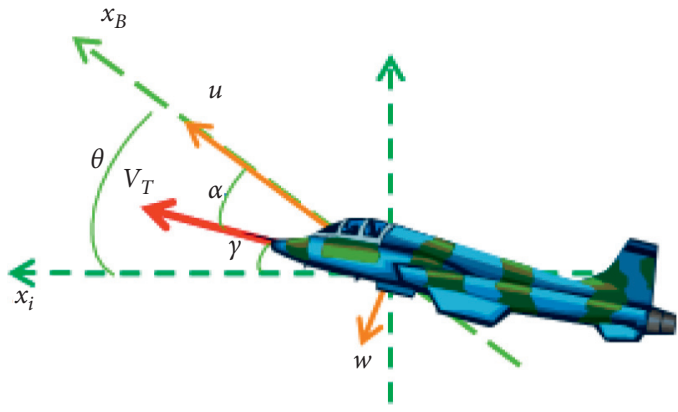

Figure 1: Model of longitudinal motion of the aircraft.

The objective is to design a backstepping sliding mode control law for the lower-triangular flight dynamics ((33)-(35) such that the flight-path angle $(\gamma)$ tracks the command $\left(\gamma_{\text {ref }}\right)$ with asymptotic stability. The performance specifications of the system should achieve wellbehaved command tracking with zero or small acceptable overshoot.

3.2. Derivation of Backstepping Sliding Mode Control Law. With state variables $(\gamma, \theta, q)$ and control input $\delta_{E}$, systems (33)-(35) are exactly the ones in (1)-(3) with $f_{1}(\gamma)=-\left(g / V_{T}\right) \cos \gamma+\widehat{L}_{0}-\widehat{L}_{\alpha} \gamma, f_{2}(\gamma, \theta)=0, f_{3}(\gamma, \theta, q)$ $=\widehat{M}_{0}+\widehat{M}_{\alpha}(\theta-\gamma)+\widehat{M}_{q} q, \quad g_{1}(\gamma)=\widehat{L}_{\alpha}, \quad g_{2}(\gamma, \theta)=1$, and $g_{3}(\gamma, \theta, q)=\widehat{M}_{\delta_{E}}$. It is clear that $f_{i}(i=1,2,3)$ and $g_{i}(i=$ $1,2,3)$ are satisfying conditions in Section 2.2. Thus, there is a direct application of Theorem 1 in Section 2 or state transformations and feedbacks as

$$
\begin{aligned}
& e_{1}(t)=\gamma-\gamma_{\text {ref }}, \\
& e_{2}(t)=\theta-\alpha_{1},
\end{aligned}
$$

where a virtual feedback $\alpha_{1}$ in equation (37) is determined as in the following equation:

$$
\begin{aligned}
& \alpha_{1}=\frac{1}{\widehat{L}_{\alpha}}\left\{-p_{1} \tanh \left[\lambda\left(\gamma-\gamma_{\text {ref }}\right)\right]+g_{p} \cos (\gamma)-\widehat{L}_{0}+\widehat{L}_{\alpha} \gamma\right\}, \\
& e_{3}(t)=q-\alpha_{2},
\end{aligned}
$$

where virtual feedback $\alpha_{2}$ in equation (39) is determined as in the following equations:

$$
\begin{aligned}
& \alpha_{2}=-p_{2} \tanh \left(\lambda\left(\theta-\alpha_{1}\right)\right)+\dot{\alpha}_{1}, \\
& \dot{\alpha}_{1}=\frac{1}{\widehat{L}_{\alpha}}\left\{-p_{1}\left(\operatorname{sech}^{2}\left[\lambda\left(\gamma-\gamma_{\mathrm{ref}}\right)\right] \lambda F_{\gamma}-g_{p} \sin (\gamma) F_{\gamma}+\widehat{L}_{\alpha} F_{\gamma}\right)\right\},
\end{aligned}
$$

$F_{\gamma}=-g_{p} \cos \gamma+\widehat{L}_{0}-\widehat{L}_{\alpha} \gamma+\widehat{L}_{\alpha} \theta$.

In addition, a BSMC law for systems (33)-(35) can be achieved in the following equation: 


$$
\begin{aligned}
\delta_{E}= & \delta_{E_{0}}+\frac{\pi}{180} \frac{1}{\widehat{M}_{\delta_{E}}}\left\{-p_{3} \tanh \left(\lambda\left(q-\alpha_{2}\right)\right)\right. \\
& \left.-\widehat{M}_{0}-\widehat{M}_{\alpha}(\theta-\gamma)-\widehat{M}_{q} q+\dot{\alpha}_{2}\right\}
\end{aligned}
$$

where $\alpha_{2}$ is determined in (40) and $\dot{\alpha}_{2}$ is determined in equations (44)-(46). The achieved BSMC law in equation (43) is used for further numerical study in the next section.

$$
\begin{aligned}
\dot{\alpha}_{2}= & -p_{2} \lambda\left(q-\dot{\alpha}_{1}\right) \operatorname{sech}^{2}\left(\lambda\left(\theta-\alpha_{1}\right)\right)+\ddot{\alpha}_{1}, \\
\ddot{\alpha}_{1}= & \frac{1}{\widehat{L}_{\alpha}}\left\{-p_{1} \operatorname{sech}^{2}\left(\lambda\left(\theta-\alpha_{1}\right)\right) \lambda \dot{F}_{\gamma}-2 p_{1} \tanh \left(\lambda\left(\theta-\alpha_{1}\right)\right)\right. \\
& \left.\cdot \operatorname{sech}\left(\lambda\left(\theta-\alpha_{1}\right)\right) \lambda^{2} F_{\gamma}^{2}-g_{p} \sin (\gamma) \dot{F}_{\gamma}-g_{p} \cos (\gamma) F_{\gamma}^{2}+\widehat{L}_{\alpha} \dot{F}_{\gamma}\right\}, \\
\dot{F}_{\gamma}= & g_{p} \sin (\gamma) F_{\gamma}+\widehat{L}_{\alpha}\left(q-F_{\gamma}\right) .
\end{aligned}
$$

By substituting the BSMC in equation (43) into systems (33)-(35), a closed-loop system is achieved for numerical simulation. Figure 2 shows a control structure design for implementing a numerical simulation. A further explanation on this block diagram can be found in [13].

\subsection{Numerical Simulation of Flight-Path Angle of F-16 Air-} craft Model. For a further verification of advance and robustness of the proposed method, a full nonlinear F-16 aircraft model or a true model is used for numerical solutions. The aerodynamic data of the F-16 aircraft model are provided in [22] in which data are achieved from low-speed static and dynamic wind-tunnel tests at the NASA Langley Research Center. In this paper, the aircraft is assumed to be in level fight at Mach 0.5 and at an altitude of $25000 \mathrm{ft}$. Based on this assumption, control surfaces, aerodynamic coefficients, and other state variables at trim condition or level flight can be calculated for further simulation. For simplicity, those numbers are not listed in this paper. A closed-loop system with the BSMC law in equation (43) and a control structure in Figure 2 are used for four different flight-path command profiles away from the trim condition as follows.

Profile 1: a flight-path angle command of 10 (deg) is applied for 5 seconds; Profile 2: a multistep command is applied for the flight-path angle, in which $\gamma_{\text {ref }}$ is put at 5 (deg) for the first 2.5 seconds, at 10 (deg) for the next 2.5 seconds, and then 5 (deg) in 5 seconds; Profile 3: a similar multistep command as in Profile 2 is applied for flight-path angle, but aircraft speed is varied from $600 \mathrm{ft} / \mathrm{s}$ to $500 \mathrm{ft} / \mathrm{s}$ during the simulation time; Profile 4: a doublet-step command is applied for flight-path angle for 10 seconds with change of aircraft center in which $\bar{x}_{\mathrm{cm}}=0.3$ is used in control design, but $\bar{x}_{\mathrm{cm}}=0.35$ will be used for this case for testing robustness. In the simulation, optimal positive gains of the BSMC in (43) $p_{1}=2.134 ; p_{2}=2.213 ; p_{3}=2.432$ are determined from the design model by using the modified genetic algorithm (MGA) [23]. The value $\lambda=3$ is selected by the try and error technique to have a good performance. Numerical results are simulated both within and without the presence of disturbances. Also, numerical results from the BSMC-based method will be compared to the integratorbackstepping control (IBSC) method [13].

3.3.1. Profile 1: Single-Step Command of Flight-Path Angle. Solid line and dash line in Figure 3 show the time response of flight-path angle of the IBSC-based control and BSMCbased control methods, respectively. Both methods can provide a well-tracking command. Results show that the settling time $t_{s}=2$ seconds of BSMC is shorter than that of the IBSC of $t_{s}=3.5$ seconds. This has been predicted in [13] due to an introduction of an integrator of the tracking error. This is a limitation of the IBSC-based control system for a high-performance aircraft and is improved by the proposed BSMC-based method. Another advance of the proposed method is that control parameters are reduced from 4 $\left(c_{0}, c_{1}, c_{2}, c_{3}\right)[13]$ to $3\left(p_{1}, p_{2}, p_{3}\right)$. This will make MGA more robust as searching for optimal control parameters. Solid line and dash line in Figure 4 show the time response of the elevator surface of the IBSC-based control and BSMCbased control methods, respectively. Results indicate that the control signal from IBSC is saturated at an initial period, and this is a reason why there is a decrease in slope for rising time of time response after that. In a similar input signal of the BSMC, a saturation occurs at $t=1.5$ seconds and leads to a decrease in magnitude of flight-path angle after that. However, saturation occurs in a short time, and thus the command tracking achieves quickly in this case. Also, results in Figures 3 and 4 indicate there is no chattering problem in the proposed control method. This is also an advance as compared to a traditional sliding mode control method [14].

3.3.2. Profile 2: Multistep Command of Flight-Path Angle. Figure 5 shows time response of flight-path angle with multistep command. The dot-dash line is the flight-path angle command, the dash line is associated with the IBSC-based control method, and the solid line is associated with the BSMC-based control methods. Results indicate the IBSC method results in a poor and less robust performance for a 


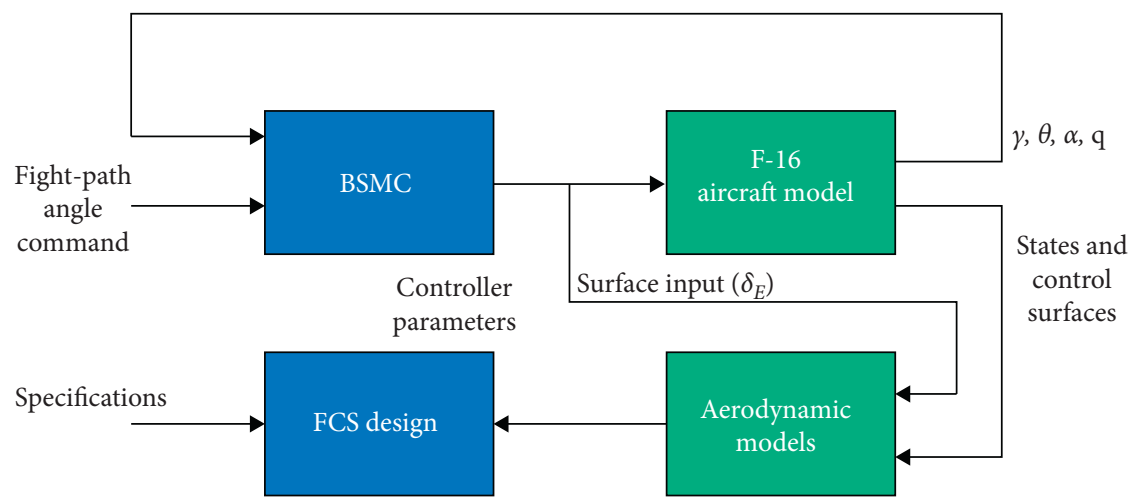

FIGURE 2: Block diagram of control architecture design.

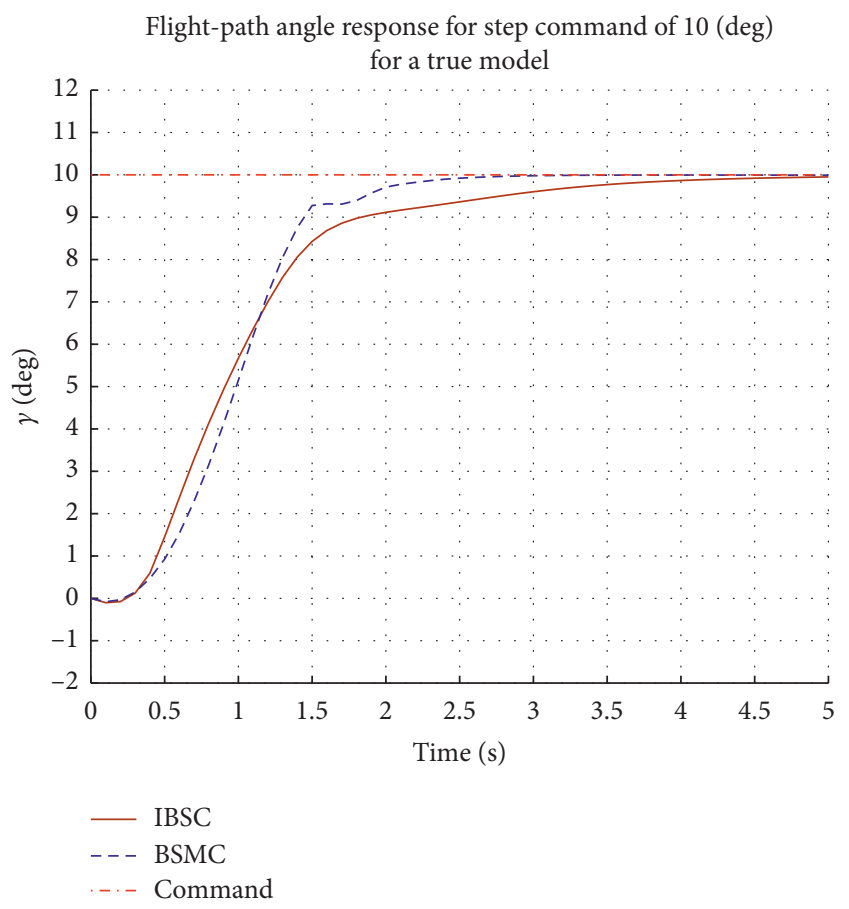

Figure 3: Time response of flight-path angle with step command 10 (deg).

short period of time, but the BSMC can provide a much better performance than that of the IBSC. Solid line and dash line in Figure 6 show the time response of the elevator surface of the IBSC-based control and BSMC-based control methods, respectively. In order to meet demand of a given performance specification, the control signal of the IBSC tends to generate a high magnitude in the negative side, but the behavior of the BSMC is relatively different. We can see that BSMC-based response of the elevator surface is more effective and suitable to the practical observation. This is predicted in Section 1, in which a suitable control gain might not able to adapt with the change in internal dynamics of the system.

3.3.3. Profile 3: Multistep Command of Flight-Path Angle with a Varying Speed of Aircraft. In order to verify the robust performance, a multistep command is applied for

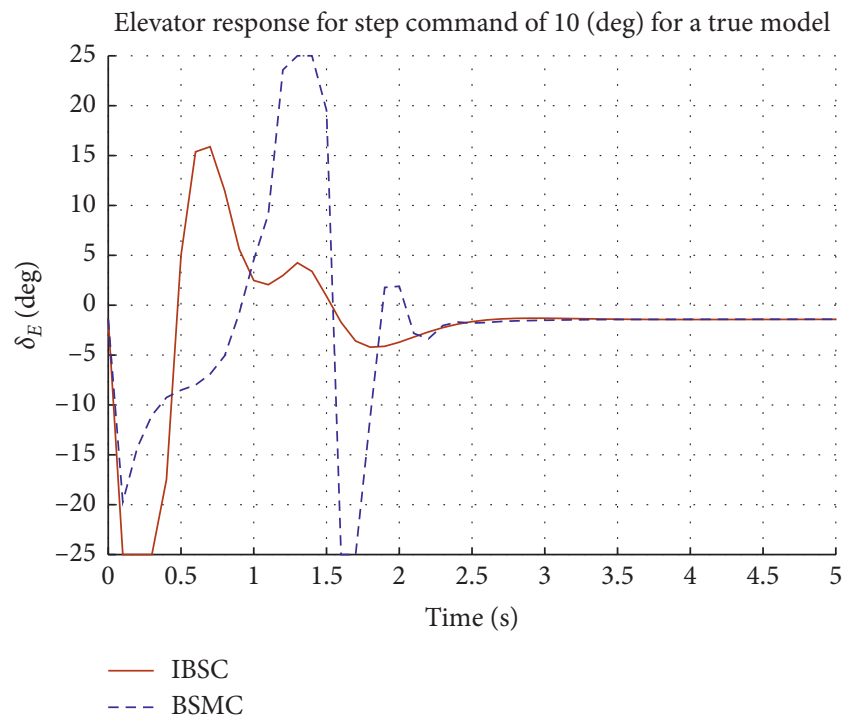

FIgURE 4: Time response of elevator surface with step command 10 (deg).

flight-path angle with a change in aircraft speed that is varied from $600 \mathrm{ft} / \mathrm{sec}$ to $500 \mathrm{ft} / \mathrm{sec}$ during the 10 seconds in simulation. In this simulation condition, dash line in Figure 7 indicates the BSMC control method which can provide a good tracking for a short period. On the other hand, solid line in Figure 7 indicates the IBSC control method which can provide a good tracking for a phugoid (or longer) period of the aircraft system. This is due to a considerable effect of the integrator to improve the tracking as time increases.

3.3.4. Profile 4: Doublet Command of Flight-Path Angle with a Variation of Aircraft Mass Center, $\bar{x}_{c m}$. In previous simulations, the mass center of the aircraft $\bar{x}_{\mathrm{cm}}=0.3$ is used for designing control parameters and in-flight simulation. In reality, the mass center might be changed due to the unload of cargoes or other equipment. Thus, a variation of this parameter can further verify reliability and robustness of the control methods. In this simulation condition, the mass center is assigned to be $\bar{x}_{\mathrm{cm}}=0.35$. Figure 8 shows time response of flight-path angle for a doublet command in the presence of mass center change. The dash line again 


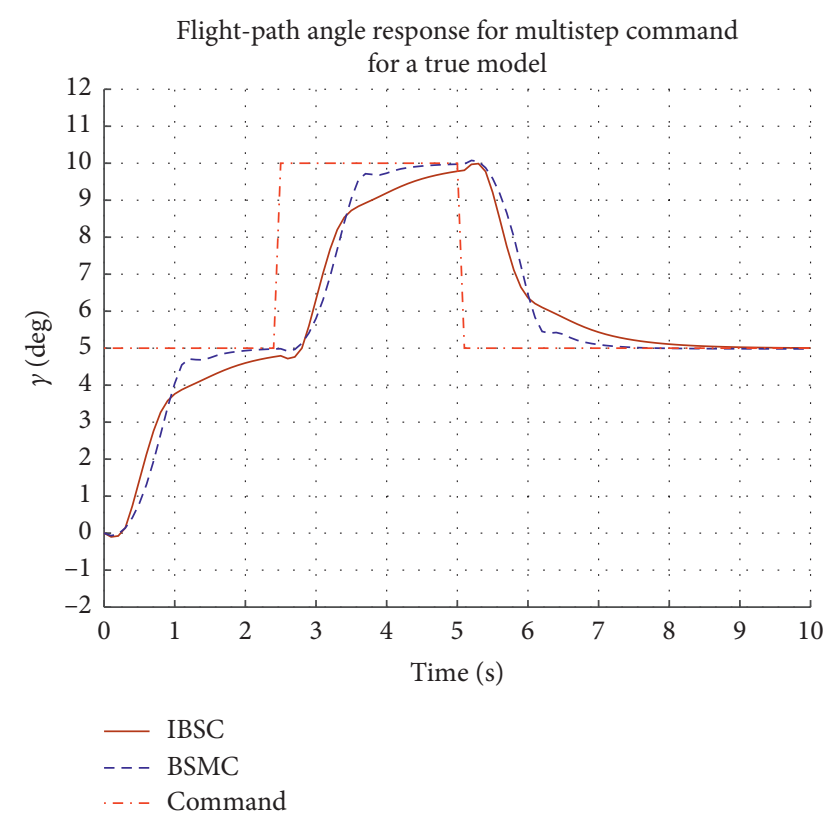

Figure 5: Time response of flight-path angle with multiple step command.

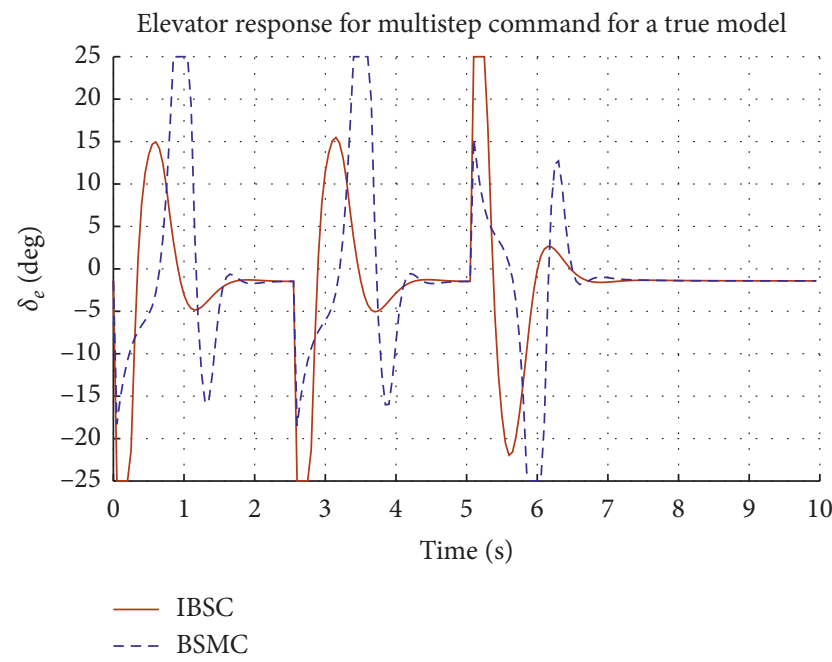

Figure 6: Time response of the elevator surface with multiple step command of flight-path angle.

indicates that the BSMC results in a well-behaved command tracking and fast response with no or small acceptable overshoot.

\section{Conclusion and Future Work}

A robust and fast-response control system plays an important role in designing and developing a high-performance aircraft. This requires an advanced control design method for nonlinear flight dynamic systems. In this paper, a hybrid robust control design method is proposed for a third-order SISO lower-triangular model of nonlinear dynamic systems in the presence of disturbances. Then, the application of flight-path angle control corresponding to the longitudinal dynamics of

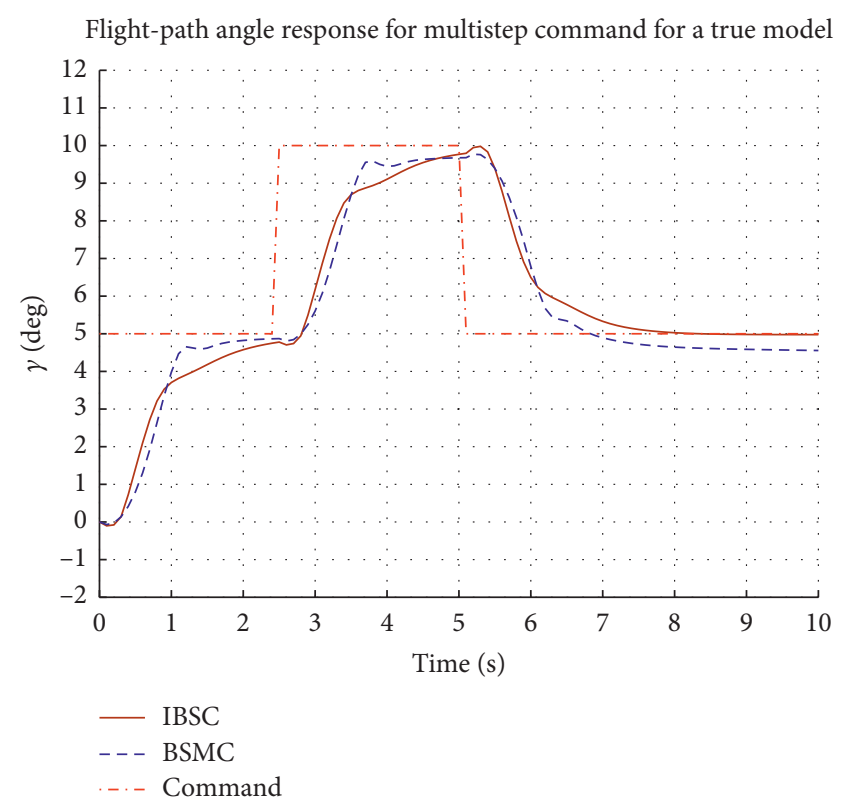

FIgURE 7: Time response of flight-path angle in time-varying change of speed of the aircraft.

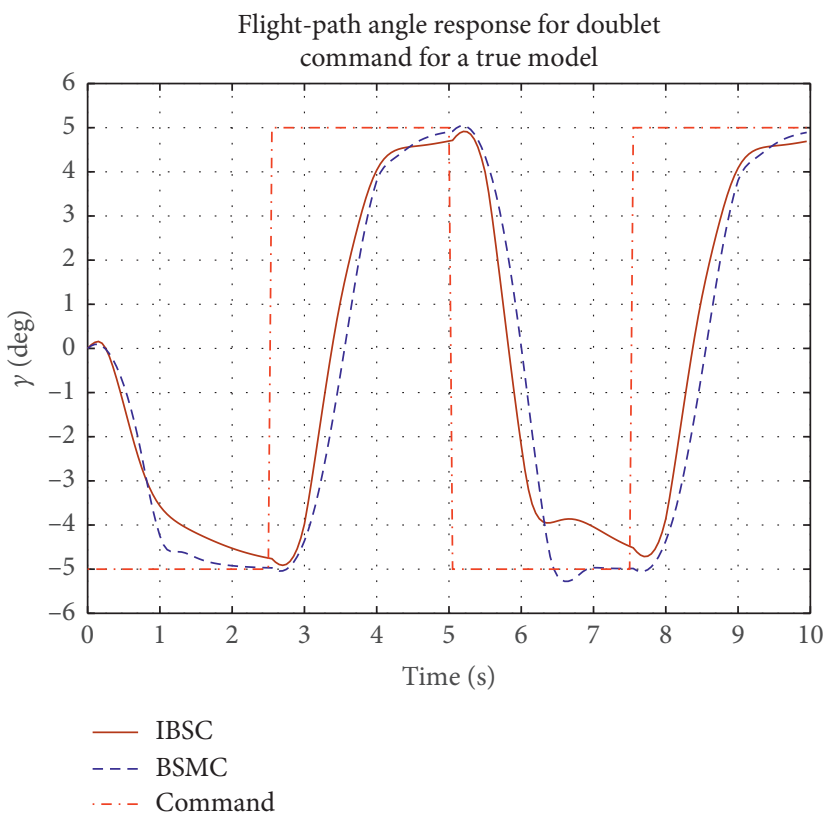

FIgURE 8: Time response of flight-path angle in doublet-step command in variation of aircraft mass center.

a high-performance F-16 aircraft simulation model is implemented. The contributions of this paper are as follows:

(i) The proposed control method also provides a diversity of nonlinear control design tools to a specific class of nonlinear dynamic systems and has a high potential for other application in engineering systems. 
(ii) A novel controller synthesis method, called backstepping sliding mode control (BSMC) law, is presented systematically for a third-order SISO lower-triangular system. The BSMC-based control method results in an advanced control system which can provide an excellent and robust performance with asymptotic stability in the presence of uncertainties and eliminate completely a chattering phenomenon that is a drawback of a traditional SMC.

(iii) A successful application is implemented for the nonlinear flight dynamic system. In particular, a BSMC for flight-path angle control of the F-16 aircraft model is derived. Then, a control algorithm is provided for in-flight closed-loop simulation. Numerical results indicate the BSMC can provide a robust performance and stability with different flight profiles and presence of external disturbances.

With the development of high-performance digital computer, robust nonlinear control methods, and computational software, the nonlinear control system will result in a considerable role in improving accuracy, flexibility, and robustness in control systems.

The field of the research focuses only on a lower-triangular model of nonlinear dynamic systems. Therefore, an extended version of a generalized form of the nonlinear dynamic system can prove a better solution. Also, application is limited in aircraft motion in longitudinal axis and simulation results neglect effectiveness of lateral dynamics of the aircraft. Thus, investigation of full nonlinear aircraft fight dynamics should be considered to validate further the proposed method. In addition, this proposed control method can be applied to other engineering systems.

\section{Data Availability}

Readers can access the data supporting the conclusions in case readers would like to repeat the results, and data can be requested by sending an e-mail to the corresponding author.

\section{Conflicts of Interest}

The authors declare that there are no conflicts of interest regarding the publication of this paper.

\section{Acknowledgments}

This work was supported by Vietnamese-German University.

\section{References}

[1] M. Krstic, P. V. Kokotovic, and I. Kanellakopoulos, Nonlinear and Adaptive Control Design, John Wiley \& Sons, Hoboken, NJ, USA, 1st edition, 1995.

[2] S. H. Lane and R. F. Stengel, "Flight control design using nonlinear inverse dynamics," Automatica, vol. 24, no. 4, pp. 471-483, 1988.
[3] L. Sonneveldt, Q. P. Chu, and J. A. Mulder, "Nonlinear flight control design using constrained adaptive backstepping," Journal of Guidance, Control, and Dynamics, vol. 30, no. 2, pp. 322-336, 2007.

[4] J. Farrell, M. Sharma, and M. Polycarpou, "Backsteppingbased flight control with adaptive function approximation," Journal of Guidance, Control, and Dynamics, vol. 28, no. 6, pp. 1089-1102, 2005.

[5] C. I. Marrison and R. F. Stengel, "Design of robust control systems for a hypersonic aircraft," Journal of Guidance, Control, and Dynamics, vol. 21, no. 1, pp. 58-63, 1998.

[6] Q. Wang and R. F. Stengel, "Robust nonlinear control of a hypersonic aircraft," Journal of Guidance, Control, and Dynamics, vol. 23, no. 4, pp. 577-585, 2000.

[7] Q. Wang and R. F. Stengel, "Robust nonlinear flight control of a high-performance aircraft," IEEE Transactions on Control Systems Technology, vol. 13, no. 1, pp. 15-26, 2005.

[8] F. Giri, A. Rabeh, and F. Ikhouane, "Backstepping adaptive control of time-varying plants," Systems \& Control Letters, vol. 36, no. 4, pp. 245-252, 1999.

[9] K.-H. Choi, T. Tran, and D.-S. Kim, "Back-stepping controller based web tension control for roll-to-roll web printed electronics system," Journal of Advanced Mechanical Design, Systems, and Manufacturing, vol. 5, no. 1, pp. 7-21, 2011.

[10] T. T. Tran, "Feedback linearization and backstepping: An equivalence in control design of strict-feedback form," IMA Journal of Mathematical Control and Information, 2019.

[11] T. T. Tran and B. Newman, "Back-stepping based flight path angle control algorithm for longitudinal dynamics," in Proceedings of the AIAA Guidance, Navigation, and Control Conference, Minneapolis, MN, USA, 2012.

[12] T. T. Tran and O. R. Gonzalez, "Backstepping-based control methodology for aircraft roll dynamics," Proceedings of the Institution of Mechanical Engineers, Part I: Journal of Systems and Control Engineering, vol. 234, no. 4, pp. 566-574, 2020.

[13] T. T. Tran and B. Newman, "Integrator-backstepping control design for nonlinear flight system dynamics," in Proceedings of the AIAA Guidance, Navigation, and Control Conference, Kissimmee, FL, USA, January 2015.

[14] J.-J. E. Slotine, "Sliding controller design for non-linear systems," International Journal of Control, vol. 40, no. 2, pp. 421-434, 1984.

[15] H. Xu, M. D. Mirmirani, and P. A. Ioannou, "Adaptive sliding mode control design for a hypersonic flight vehicle," Journal of Guidance, Control, and Dynamics, vol. 27, no. 5, pp. 829838, 2004.

[16] B. Wu, D. Wang, and E. K. Poh, "Decentralized sliding-mode control for attitude synchronization in spacecraft formation," International Journal of Robust and Nonlinear Control, vol. 23, no. 11, pp. 1183-1197, 2013.

[17] S. Seshagiri and E. Promtun, "Sliding mode control of F-16 longitudinal dynamics," in Proceedings of the American Control Conference, pp. 1770-1775, Seattle, WA, USA, June 2008.

[18] H. Alwi, C. Edwards, and M. T. Hamayun, "Nonlinear integral sliding mode fault tolerant longitudinal aircraft control," in Proceedings of the IEEE International Conference on Control Applications (CCA), pp. 970-975, Denver, CO, USA, September 2011.

[19] E. Promtun and S. Seshagiri, "Sliding mode control of pitchrate of an F-16 aircraft," IFAC Proceedings Volumes, vol. 41, no. 2, pp. 1099-1104, 2008. 
[20] Y. Wang, Y. Xia, H. Li, and P. Zhou, "A new integral sliding mode design method for nonlinear stochastic systems," Automatica, vol. 90, pp. 304-309, 2018.

[21] J. Fei and Z. Feng, "Fractional-order finite-time supertwisting sliding mode control of micro gyroscope based on double-loop fuzzy neural network," IEEE Transactions on Systems, Man, and Cybernetics: Systems, pp. 1-15, 2020.

[22] L. T. Nguyen, M. E. Ogburn, W. P. Gilbert, K. S. Kibler, P. W. Brown, and P. L. Deal, "Simulator study of stall/poststall characteristics of a fighter airplane with relaxed longitudinal static stability," Techical Report L-12854, NASA, Washington, DC, USA, 1979.

[23] K. H. Choi, T. T. Thanh, Y. B. Su, and D. S. Kim, "An new approach for intelligent control system design using the modified genetic algorithm," International Journal of Intelligent Systems Technologies and Applications, vol. 9, no. 3/4, pp. 300-315, 2010. 